\title{
Arc Spaces and Rogers-Ramanujan Identities
}

\author{
Clemens Bruschek 征 Hussein Mourtada ${ }^{2}$ and Jan Schepers 非
}

${ }^{1}$ University of Vienna, Vienna, Austria

${ }^{2}$ Laboratoire de Mathématiques de Versailles, Versailles, France

${ }^{3}$ K. U. Leuven, Leuven, Belgium

\begin{abstract}
Arc spaces have been introduced in algebraic geometry as a tool to study singularities but they show strong connections with combinatorics as well. Exploiting these relations we obtain a new approach to the classical RogersRamanujan Identities. The linking object is the Hilbert-Poincare series of the arc space over a point of the base variety. In the case of the double point this is precisely the generating series for the integer partitions without equal or consecutive parts.

Résumé. Les espaces des arcs ont été introduit pour étudier les singularités, mais ils ont aussi un lien fort avec la combinatoire. Ce lien permet une nouvelle approche vers les identités de Rogers-Ramanujan. L'objet permettant cette approche est la série de Hilbert-Poincaré de l'algèbre des arcs centrés en un point de la variété de base. Dans le cas où cette variété est le point double, cette série est la série génératrice des partitions d'un nombre entier sans parties égales ou consécutives.

Resumen. Los espacios de arcos han sido introducidos en geometría algebraica como una herramienta para estudiar singularidades, sin embargo también han mostrado una robusta conexión con la combinatoria. Exprimiendo estas relaciones obtenemos un nuevo enfoque de las identidades de Rogers-Ramanujan. El objeto vinculante son las series de Hilbert-Poincaré de los espacios de arcos en un punto de la variedad base. En el caso del punto doble estas series son precisamente las series generadoras de las particiones enteras sin partes iguales o consecutivas.
\end{abstract}

Keywords: formal power series, Hilbert-Poincaré series, partitions, Rogers-Ramanujan Identities, arc spaces, infinite dimensional Gröbner basis

\section{Introduction}

Arc spaces have first appeared in the work of John Nash (see Nash (1995)) to study resolution of singularities of algebraic varieties. Besides their geometric usefulness (see Ein and Mustaţă (2004), and Ishii (2007) for an overview) arc spaces show strong relations with combinatorics. In this extended abstract we indicate how to exploit this connection both for algebraic as well as combinatorial benefit. Especially, we give a new approach towards the well-known Rogers-Ramanujan identities via these ideas. An extended version of this abstract including complete proofs can be found in Bruschek et al. (2011).

\footnotetext{
${ }^{\dagger}$ Austrian Science Fund (FWF): P21461, I382

$\ddagger$ Postdoctoral Fellow of the Research Foundation - Flanders (FWO) 
Before starting with an overview of this note let us emphasize the main algebraic and combinatorial aspects presented here. First, we suggest to study algebro-geometric properties of algebraic (or analytic) varieties via natural Hilbert-Poincaré series attached to arc spaces. Second, we propose to derive identities between partitions by looking at suitable ideals in a polynomial ring in countably many variables endowed with a natural grading. Connecting both ideas will demand handling Gröbner basis in countably many variables, a problem which has been successfully dealt with in different contexts over the last years (see Hillar and Sullivant (2009), Draisma (2010)). In the present situation - that is for very specific ideals - salvation from the natural obstruction of being infinitely generated comes in the shape of a derivation making the respective ideals differential.

We start in Section 2 with the definition of the arc space of an algebraic variety as the set of formal power series solutions (in one variable) to the defining equations of the variety. This data can be encoded in conditions on the coefficients of the power series, thus yielding countably many equations in countably many variables. It turns out that these equations have very nice properties: they are homogeneous with respect to a grading which endows the $i$ th coefficient of the power series with 'weight' $i$, and they are generated from the defining equations by applying a specific derivation (which will be introduced in the proof of Lemma 4.4p. The first property involves that we can consider the coordinate algebra of the arc space as a graded algebra and especially we can try to compute its corresponding Hilbert-Poincaré series. This is elaborated in Section 3 where we also encounter a classical combinatorial object: partitions. These naturally arise when computing weights of monomials. Indeed, a monomial $y_{1}^{\alpha_{1}} \cdots y_{j}^{\alpha_{j}}$ has weight $\alpha_{1} \cdot 1+\cdots+\alpha_{j} \cdot j$. Asking for the number of monomials (up to coefficients) of some weight $m$ is thus asking for the number of partitions of $m$. In Section 4- using a well-known result from the theory of partitions - we are able to compute the Hilbert-Poincaré series of a simple, though already interesting, algebraic variety. On the other hand, we can use standard techniques from commutative algebra to compute the Hilbert-Poincaré series for the double point (i.e., the algebraic variety given by one polynomial equation $y^{2}=0$ in one variable $y$ ), thus retrieving the Rogers-Ramanujan identities. Using triple or even $n$-fold points we would obtain Gordon's generalizations of the Rogers-Ramanujan identities (see Andrews (1998) for a precise statement of those). We conclude with a short synopsis of the theory of Hilbert-Poincaré series.

The first named author expresses his gratitude to Georg Regensburger and Josef Schicho for many useful discussions on these topics; the second author thanks Monique Lejeune-Jalabert for introducing him to Hilbert-Poincaré series; the third named author would like to thank Johannes Nicaise for explaining him several facts about arc spaces in detail. All authors are indebted to an anonymous referee for pointing out the connection between Lie algebras and the Rogers-Ramanujan Identities.

\section{Arc spaces}

Let us briefly recall the notion of arc space. Essentially arc spaces are sets of solutions to polynomial equations in a formal power series ring in one indeterminate. To be more precise, let $f \in k\left[x_{1}, \ldots, x_{n}\right]$ be a polynomial in $n$ variables $x_{1}, \ldots, x_{n}$ with coefficients in a field $k$. The formal power series ring in one variable $t$ over the field $k$ is written as $k[[t]]$. The arc space of the algebraic variety $X$ defined by $f$ is the set of power series solutions $x(t)=\left(x_{1}(t), \ldots, x_{n}(t)\right) \in k[[t]]^{n}$ to the equation $f(x(t))=0$. We denote it by $X_{\infty}$. This set turns out to be eventually algebraic in the sense that it is given by polynomial equations 
(though there are countably many of them). Indeed, write $x_{j}(t)=\sum_{i=0}^{\infty} x_{j}^{(i)} t^{i}$ with new variables $x_{j}^{(i)}$, $1 \leq j \leq n$ and $i \in \mathbb{N}=\{0,1,2, \ldots$,$\} . Expanding f(x(t))$ as a power series in $t$ gives

$$
f(x(t))=F_{0}+F_{1} t+F_{2} t^{2}+\cdots
$$

with $F_{i} \in k\left[x_{j}^{(i)} ; 1 \leq j \leq n, i \in \mathbb{N}\right]$ polynomials in the coefficients of $t$ in $x(t)$ (see Bruschek (2010) or Bruschek (2009) for more combinatorial properties of these polynomials). Therefore, a given vector of formal power series $a(t) \in k[[t]]^{n}$ is an element of the arc space $X_{\infty}$ if and only if its coefficients $a_{j}^{(i)}$ fulfill the equations $F_{0}, F_{1}, \ldots$. Algebraically the corresponding set of solutions is described by its coordinate algebra

$$
J_{\infty}(X)=k\left[x_{j}^{(i)} ; 1 \leq j \leq n, i \in \mathbb{N}\right] /\left(F_{0}, F_{1}, \ldots\right) .
$$

Note, that the equation $F_{0}$ is nothing but our original polynomial $f$ written in variables $x_{j}^{(0)}, 1 \leq j \leq n$. We will mostly be interested in the case where we substitute in the $F_{i}$ for $\left(x_{1}^{(0)}, \ldots, x_{n}^{(0)}\right)$ an $n$-tuple $p=\left(p_{1}, \ldots, p_{n}\right)$ which fulfills $f(p)=0$, i.e., which describes a point on our algebraic variety $X$. The resulting algebra is called the focussed arc algebra and denoted by $J_{\infty}^{p}(X)$. Without loss of generality we may assume that $p$ is the origin. If we write $f_{i}$ for the polynomial we obtain after substituting 0 for all $x_{j}^{(0)}$ in $F_{i}$ then

$$
J_{\infty}^{0}(X)=k\left[x_{j}^{(i)} ; 1 \leq j \leq n, i \geq 1\right] /\left(f_{1}, f_{2}, \ldots\right) .
$$

For sake of completeness we introduce also the jet spaces of $X$. These are usually defined as truncated power series solutions to the defining equations of the variety. In the present situation the mth jet space $X_{m}$ of $X$ is

$$
X_{m}=\left\{x(t) \in\left(k[[t]] /(t)^{m+1}\right)^{n} ; f(x(t))=0 \bmod (t)^{m+1}\right\} .
$$

Its coordinate algebra is simply given by

$$
J_{m}(X)=k\left[x_{j}^{(i)} ; 1 \leq j \leq n, 0 \leq i \leq m\right] /\left(F_{0}, F_{1}, \ldots, F_{m}\right) .
$$

with $F_{i}$ as introduced above. Accordingly, we can also define the focussed $m$ th jet space as

$$
J_{m}^{0}(X)=k\left[x_{j}^{(i)} ; 1 \leq j \leq n, 1 \leq i \leq m\right] /\left(f_{1}, f_{2}, \ldots, f_{m}\right)
$$

It is obvious how these notions extend to the case that our base variety $X$ is not given by one polynomial $f$ but by finitely many of them. For simplicity of notation we will restrict our considerations to the hypersurface case though.

\section{The Hilbert Poincaré series of an arc algebra}

Let $X$ be the algebraic variety defined by a polynomial $f \in k\left[x_{1}, \ldots, x_{n}\right]$ with $f(0)=0$ and $J_{\infty}(X)$ respectively $J_{\infty}^{0}(X)$ the corresponding arc algebra respectively focussed arc algebra (at 0 ). Both are quotient algebras of the polynomial ring $k\left[x_{j}^{(i)} ; 1 \leq j \leq n, i \in \mathbb{N}\right]$. Besides its natural grading via the classical 'degree' we will endow this polynomial ring with a grading induced by the following weight: $\mathrm{wt}\left(x_{j}^{(i)}\right)=i$. 
Proposition 3.1 The ideal $\left(f_{1}, f_{2}, \ldots\right) \subseteq k\left[x_{j}^{(i)} ; 1 \leq j \leq n, i \in \mathbb{N} \backslash\{0\}\right]$ is homogeneous with respect to the weight $\mathrm{wt}$; hence the focussed arc algebra $J_{\infty}^{0}(X)$ is a graded algebra. Moreover, all homogeneous parts $J_{\infty}^{0}(X)_{i}$ of weight $i$ are finite dimensional $k$-vector spaces.

Remark 3.2 The property that $f_{i}$ is homogeneous with respect to wt is inherited from $F_{i}$ which is homogeneous with respect to wt as well.

Having a graded $k$-algebra with finite dimensional homogeneous components it is natural to ask for the corresponding Hilbert-Poincaré series. This is the generating series for the sequence of dimensions

$$
\operatorname{dim}_{k} J_{\infty}^{0}(X)_{i}, i \in \mathbb{N} .
$$

For a short synopsis of technical results in the theory of Hilbert-Poincaré series we refer to the Appendix, Section 5, or a text on commutative algebra like Greuel and Pfister (2002). In the remaining sections we will refer to Hilbert-Poincare series in short as HP-series. The HP-series of the focussed arc algebra $J_{\infty}^{0}(X)$ will be denoted by $\operatorname{HP}_{J_{\infty}^{0}(X)}(t)$.

Example 3.3 As a first example we compute the HP-series of the arc space of the affine line $\mathbb{A}^{1}$ focussed at 0 . The affine line has coordinate ring $k[y]$, thus, $J_{\infty}^{0}\left(\mathbb{A}^{1}\right)=k\left[y_{1}, y_{2}, \ldots\right]$. The mth homogeneous piece of $J_{\infty}^{0}\left(\mathbb{A}^{1}\right)$ with respect to wt is the $k$-vector space spanned by all monomials of weight $m$. Let $y^{\alpha}$ with $\alpha \in \mathbb{N}^{(\mathbb{N})}$ denote a monomial in $k\left[y_{1}, y_{2}, \ldots\right]$. Clearly, it has weight m exactly if

$$
\alpha_{1} \cdot 1+\cdots+\alpha_{m} \cdot m=m .
$$

Note, that no $y_{i}$ with $i>m$ can appear in $y^{\alpha}$ since then the weight of the monomial would exceed $m$. Equation (1) shows that every monomial of weight $m$ corresponds to a partition of $m$, i.e.,

$$
\operatorname{dim}_{k} J_{\infty}^{0}\left(\mathbb{A}^{1}\right)_{m}=p(m)
$$

where $p: \mathbb{Z} \rightarrow \mathbb{Z}$ denotes the partition function mapping every integer $m$ to its number of partitions. Therefore we obtain (see Andrews (1998)):

$$
\operatorname{HP}_{J_{\infty}^{0}\left(\mathbb{A}^{1}\right)}(t)=\prod_{i=1}^{\infty} \frac{1}{1-t^{i}} .
$$

Henceforth we will denote the HP-series computed in the last example by $\mathbb{H}$. It is not hard to prove that for the focussed arc algebra of affine $n$-space $\mathbb{A}^{n}$ the following is true

Proposition 3.4 With the above introduced notation we obtain

$$
\operatorname{HP}_{J_{\infty}^{0}\left(\mathbb{A}^{n}\right)}(t)=\mathbb{H}^{n} .
$$

Remark 3.5 For the more algebro-geometric inclined reader we note that if $X$ and $Y$ are analytically isomorphic at $p \in X$ and $q \in Y$ then the corresponding focussed arc algebras are isomorphic as graded algebras, thus having the same HP-series. Therefore, Proposition 3.4 also gives the HP-series for a smooth point of an arbitrary $n$-dimensional variety. 
Remark 3.6 Recall that the multiplicity or order of $f \in k\left[x_{1}, \ldots, x_{n}\right]$ is defined as the minimal degree of a monomial appearing in $f$, i.e.,

$$
\operatorname{ord} f=\min \{|\alpha| ; \alpha \in \operatorname{supp}(f)\},
$$

where we define $|\alpha|=\alpha_{1}+\cdots+\alpha_{n}$ as usual. Moreover, we consider the truncation operator

$$
\tau_{\leq r}: k[[t]] \rightarrow k[t]: \sum_{i \geq 0} a_{i} t^{i} \mapsto \sum_{i=0}^{r} a_{i} t^{i} .
$$

As one would expect

$$
\tau_{\leq m} \mathrm{HP}_{J_{m}^{0}(X)}(t)=\tau_{\leq m} \mathrm{HP}_{J_{\infty}^{0}(X)}(t) .
$$

From this it is not hard to show the following:

Proposition 3.7 Let $f \in k\left[x_{1}, \ldots, x_{n}\right]$ with $f(0)=0$ define the algebraic variety $X$. Then $f$ has multiplicity $r$ if and only if $r$ is the maximal number such that

$$
\tau_{\leq r-1} \mathrm{HP}_{J_{\infty}^{0}(X)}=\tau_{\leq r-1} \mathbb{H}^{n} .
$$

Moreover

$$
\tau_{\leq r} \mathrm{HP}_{J_{\infty}^{0}(X)}=\tau_{\leq r} \mathbb{H}^{n}-t^{r} .
$$

Using results of Ein and Mustatăa (2004) one can show the following, which was obtained in Mourtada (2010) by explicit computation:

Proposition 3.8 If $X$ is a surface with a rational double point at the origin then

$$
\operatorname{HP}_{J_{\infty}^{0}(X)}(t)=\left(\frac{1}{1-t}\right)^{3}\left(\prod_{i \geq 2} \frac{1}{1-t^{i}}\right)^{2} .
$$

A similar result holds for normal crossings singularities. Indeed, using Theorem 2.2 from Goward and Smith 2006) one can show:

Proposition 3.9 Let $X$ be the hypersurface given by $x_{1} \cdots x_{e}=0$, e $\leq n$, in $\mathbb{A}_{k}^{n}$. Then

$$
\operatorname{HP}_{J_{\infty}^{0}(X)}(t)=\left(\prod_{i=1}^{e-1} \frac{1}{1-t^{i}}\right)^{n}\left(\prod_{i \geq e} \frac{1}{1-t^{i}}\right)^{n-1} .
$$

\section{Rogers-Ramanujan Identities}

The Rogers-Ramanujan Identities are well-known relations between quantities of certain integer partitions. They will appear in what follows for two reasons: one can use them to compute HP-series for some varieties and in turn we are able to approach them by computing the very same HP-series by different techniques.

We will use the Rogers-Ramanujan Identities in the following form (sometimes called (first) RogersRamanujan identity in the literature). For a classical proof and an account of its history, see Andrews (1998) Chpt. 7. 
Theorem 4.1 (Rogers-Ramanujan Identity) The number of partitions of $n$ into parts congruent to 1 or 4 modulo 5 is equal to the number of partitions of $n$ into parts that are neither repeated nor consecutive.

Its analytic counterpart can be formulated as (see Corollary 7.9 in Andrews (1998)):

Corollary 4.2 (Rogers-Ramanujan Identity, analytic form) Theorem 4.1 is equivalent to the identity

$$
1+\frac{t}{1-t}+\frac{t^{4}}{(1-t)\left(1-t^{2}\right)}+\frac{t^{9}}{(1-t)\left(1-t^{2}\right)\left(1-t^{3}\right)}+\cdots=\prod_{i=1,4 \bmod 5} \frac{1}{\left(1-t^{i}\right)} .
$$

\subsection{The Hilbert-Poincaré series of the double point}

Let us first use Theorem 4.1 to compute the Hilbert-Poincaré series of the double point $X: y^{2}=0$ in $\mathbb{A}^{1}$. The corresponding focussed arc algebra looks as follows:

$$
J_{\infty}^{0}(X)=k\left[y_{1}, y_{2}, \ldots\right] /\left(2 y_{1}^{2}, 6 y_{1} y_{2}, 6 y_{2}^{2}+8 y_{1} y_{3}, \ldots\right) .
$$

As before we denote by $f_{i}, i \geq 2$, the generators of the defining ideal $I$ of $J_{\infty}^{0}(X)$. In order to compute the HP-series of this algebra it suffices to compute the HP-series of the algebra

$$
k\left[y_{1}, y_{2}, \ldots\right] / L(I)
$$

where $L(I)$ is the leading ideal of $I$ with respect to some weight-compatible monomial ordering on $k\left[y_{1}, y_{2}, \ldots\right]$, see Theorem 5.3. We endow $k\left[y_{0}, y_{1}, \ldots\right]$ (and consequently $k\left[y_{1}, y_{2}, \ldots\right]$ ) with the following monomial ordering: for $\alpha, \beta \in \mathbb{N}^{(\mathbb{N})}$ we have $y^{\alpha}>y^{\beta}$ if and only if wt $\alpha>$ wt $\beta$ or, in case of equality, the last non-zero entry of $\alpha-\beta$ is negative. The leading monomial of $f_{i}$ with respect to this ordering is determined as

Proposition 4.3 The leading monomial of $f_{i}$ is $(j \geq 1)$

$$
\operatorname{lm}\left(f_{i}\right)=\left\{\begin{array}{ll}
y_{j} y_{j+1} & i=2 j+1 \\
y_{j}^{2} & i=2 j
\end{array} .\right.
$$

From this we can derive in the present situation $L(I)$ :

Lemma 4.4 The leading ideal of $I=\left(f_{i} ; i \geq 2\right)$ is given by $\left(\operatorname{lm}\left(f_{i}\right) ; i \geq 2\right)$.

Remark 4.5 More precisely the following holds: the leading monomials of $\left(f_{2}, \ldots, f_{q}\right)$ of weight less equal $q$ are generated by $\operatorname{lm}\left(f_{i}\right), 2 \leq i \leq q$. In other words: If we extend $\left\{f_{2}, \ldots, f_{q}\right\}$ to a Gröbner basis of $\left(f_{2}, \ldots, f_{q}\right)$ all added elements will be of weight larger equal $q+1$.

Before giving a short sketch of the proof we introduce the following terminology (cf. Cox et al. (1997)): let $g \in k\left[x_{1}, \ldots, x_{n}\right]$ and let $\left(h_{1}, \ldots, h_{q}\right) \subseteq k\left[x_{1}, \ldots, x_{n}\right]$ be an ideal. We say that $g$ reduces to 0 modulo $\left(h_{1}, \ldots, h_{q}\right)$ if there exist $a_{i} \in k\left[x_{1}, \ldots, x_{n}\right], 1 \leq i \leq q$, with $g=a_{1} h_{1}+\cdots+a_{q} h_{q}$ and any leading monomial of $a_{i} h_{i}, 1 \leq i \leq q$, is less or equal to the leading monomial of $g$.

Sketch of Proof: According to the theory of Gröbner basis it suffices to show that all $S$-polynomials of the generators $f_{i}$ reduce to 0 modulo the ideal $\left(f_{2}, f_{3}, \ldots\right)$. In addition, we may restrict our considerations to $S\left(f_{i}, f_{j}\right)$ with coprime leading monomials $\operatorname{lm}\left(f_{i}\right)$ and $\operatorname{lm}\left(f_{j}\right)$ (see for example Cox et al. (1997), $\S 9$, 
Proposition 4). By Proposition 4.3 this reduces our investigation to $S$-polynomials of the following three types: $S\left(f_{2 j-1}, f_{2 j}\right), S\left(f_{2 j}, f_{2 j+1}\right)$ and $S\left(f_{2 j-1}, f_{2 j+1}\right)$. We prove that all these $S$-polynomials reduce to 0 by exploiting the differential structure of the arc ideal $F_{0}, F_{1}, \ldots$ : every $F_{i}$ can be obtained from $F_{i-1}$ by applying the $k$-derivation

$$
D: k\left[y_{0}, y_{1}, \ldots\right] \rightarrow k\left[y_{0}, y_{1}, \ldots\right]
$$

given by $D y_{i}=y_{i+1}$. Moreover, for $i \geq 2$ both $F_{i}$ and $f_{i}$ have the same leading term, thus, any $S$ polynomial of $f_{i}$ and $f_{j}$ lifts to an $S$-polynomial of $F_{i}$ and $F_{j}$; it suffices to reduce $S\left(F_{i}, F_{j}\right)$ to 0 . This can be achieved by applying an appropriate power of the derivation $D$ to the simple relation

$$
2 y_{1} F_{0}-y_{0} F_{1}=0 \text {. }
$$

To give an example, note that $S\left(F_{3}, F_{4}\right)=y_{2} F_{3}-y_{1} F_{4}$, and

$$
0=D^{4}\left(2 y_{1} F_{0}-y_{0} F_{1}\right)=2 y_{5} F_{0}+7 y_{4} F_{1}+8 y_{3} F_{2}+2 S\left(F_{3}, F_{4}\right)-y_{0} F_{5} .
$$

This shows that $S\left(F_{3}, F_{4}\right)$ reduces to 0 modulo $\left(F_{0}, F_{1}, \ldots\right)$.

Remark 4.6 The task of proving Lemma 4.4 or more generally of determining the leading ideal for the defining ideal of an arc algebra is in essence the determination of a Gröbner basis for an ideal which has countably many generators. Fortunately, the infinitely many generators are not arbitrary but determined by a finite number of polynomials using the derivation $D$ which was introduced in the above sketch of proof. Similar situations appeared in the work of Hillar and Sullivant (2009), and Draisma (2010).

The computation of the HP-series of $k\left[y_{1}, y_{2}, \ldots\right] / L(I)$ allows an easy combinatorial interpretation: the weight of a monomial $y^{\alpha}$ can be interpreted, as we have seen already in Section 3 as an integer partition. By factoring out $L(I)$ we factor out all monomials $y^{\alpha}$ which contain as factors an $y_{i}^{2}$ or $y_{i} y_{i+1}$. Therefore, the weights of the remaining monomials correspond to integer partitions without repeated or consecutive parts. Thus, from Theorem 4.1 we deduce:

Theorem 4.7 The Hilbert-Poincaré series of the focussed arc algebra $J_{\infty}^{0}(X)$ of the double line $X: y^{2}=$ 0 over the origin equals:

$$
\mathrm{HP}_{J_{\infty}^{0}(X)}(t)=\prod_{\substack{i=1,4 \\ \bmod 5}}^{\infty} \frac{1}{1-t^{i}}
$$

Remark 4.8 We have recently learned from Edward Frenkel that this result can be obtained in a completely different way, namely by studying representations of the Virasoro algebra Feigin and Frenkel (1993).

\subsection{An alternative approach to Rogers-Ramanujan}

In the previous section we used a combinatorial interpretation of the leading ideal of $I=\left(f_{2}, f_{3}, \ldots\right)$ to compute the HP-series of the corresponding graded algebra. There are commutative algebra methods to do this as well which yield a new approach to the Rogers-Ramanujan identity. By applying these we will obtain a recursion formula for the generating functions appearing therein which has already been considered by Andrews and Baxter (1989), though the present method gives a natural way to obtain it. 
Consider the graded algebra $S=k\left[y_{i} ; i \geq 1\right] / L(I)$. It is immediate (see the proof of Proposition 4.7) that its HP-series equals the generating series of the number of partitions of an integer $n$ without repeated or consecutive parts. Differently, we compute the HP-series of $S$ by recursively defining a sequence of formal power series (generating functions) in $t$ which converges in the $(t)$-adic topology to the desired HP-series. We will simply write $k[\geq d]$ for the polynomial ring $k\left[y_{i} ; i \geq d\right]$. It will be endowed with the grading wt $y_{i}=i$. The ideal generated by $y_{i}^{2}, y_{i} y_{i+1}, i \geq d$, in $k[\geq d]$ will be denoted by $I_{d}$. As usual, if $E$ is an ideal in a ring $R$ and $f \in R$ then we denote the ideal quotient, i.e.,

$$
\{a \in R ; a \cdot f \in E\}
$$

by $(E: f)$. Corollary 5.2 implies in the present situation

$$
\mathrm{HP}_{k[\geq d] / I_{d}}(t)=\mathrm{HP}_{k[\geq d+1] / I_{d+1}}(t)+t^{d} \cdot \mathrm{HP}_{k[\geq d+2] / I_{d+2}}(t) .
$$

For simplicity of notation let $h(d)$ stand for $\mathrm{HP}_{k[\geq d] / I_{d}}(t)$. Then the last equation reads as

$$
h(d)=h(d+1)+t^{d} \cdot h(d+2)
$$

and one deduces

Proposition 4.9 For the $H P$-series $\mathrm{HP}_{J_{\infty}^{0}(X)}(t)=h(1)$ we obtain

$$
h(1)=A_{d} \cdot h(d)+B_{d+1} \cdot h(d+1),
$$

for $A_{i}, B_{i} \in k[[t]]$ fulfilling the following recursion

$$
\begin{aligned}
A_{d} & =A_{d-1}+B_{d} \\
B_{d+1} & =A_{d-1} \cdot t^{d-1}
\end{aligned}
$$

with initial conditions $A_{1}=A_{2}=1$ and $B_{2}=0, B_{3}=t$.

If $\left(s_{d}\right)_{d \in \mathbb{N}}$ is a sequence of formal power series $s_{d} \in k[[t]]$ we will denote by $\lim s_{d}$ its limit - if it exists - in the $(t)$-adic topology. Since ord $B_{d} \geq d-2$ it is immediate that both $\lim A_{d}$ and $\lim B_{d}$ exist, in fact: $\lim B_{d}=0$ and

$$
h(1)=\lim A_{d} .
$$

The recursion from Proposition 4.9 can easily be simplified. We obtain:

Corollary 4.10 With the above introduced notation $\mathrm{HP}_{J_{\infty}^{0}(X)}(t)=\lim A_{d}$ where $A_{d}$ fulfills

$$
A_{d}=A_{d-1}+t^{d-2} \cdot A_{d-2}
$$

with initial conditions $A_{1}=A_{2}=1$.

The recursion appearing in this corollary is well-known since Andrews and Baxter (1989). Its limit is precisely the infinite product

$$
\prod_{\substack{i=1,4 \\ \bmod 5}}^{\infty} \frac{1}{1-t^{i}}
$$

i.e., the generating series of the number of partitions with parts equal to 1 or 4 modulo 5 . Note, that our construction gives the generating series $G_{i}$ defined in Andrews and Baxter (1989) an interpretation as Hilbert-Poincaré series of the quotients $k[\geq i] / I_{i}$. This immediately implies that the series $G_{i}$ are of the form $G_{i}=1+\sum_{j \geq i} G_{i j} t^{j}$. 


\section{Appendix}

In this section we collect some of the basics about the theory of Hilbert-Poincare series. For a detailed introduction, especially proofs, we refer to Greuel and Pfister (2002).

Let $A$ be a $\left(\mathbb{Z}\right.$-)graded $k$-algebra and let $M=\oplus_{i \in \mathbb{Z}} M_{i}$ be a graded $A$-module with $i$ th graded pieces $A_{i}$ and $M_{i}$ of finite $k$-dimension. The Hilbert function $H_{M}: \mathbb{Z} \rightarrow \mathbb{Z}$ of $M$ is defined by $H_{M}(i)=\operatorname{dim}_{k} M_{i}$, and its corresponding generating series

$$
\operatorname{HP}_{M}(t)=\sum_{i \in \mathbb{Z}} H_{M}(i) t^{i} \in \mathbb{Z}[[t]]
$$

is called the Hilbert-Poincaré series of $M$. It is well-known that if $A$ is a Noetherian $k$-algebra generated by homogeneous elements $x_{1}, \ldots, x_{n}$ of degrees $d_{1}, \ldots, d_{n}$ and $M$ is a finitely generated $A$-module then

$$
\operatorname{HP}_{M}(t)=\frac{Q_{M}(t)}{\prod_{i=1}^{n}\left(1-t^{d_{i}}\right)}
$$

for some $Q_{M}(t) \in \mathbb{Z}[t]$ which is called the (weighted) first Hilbert series of $M$. If $A$ respectively $M$ is non-Noetherian then the Hilbert-Poincaré series of $M$ need not be rational anymore. For the rest of this section we assume that the polynomial ring $k\left[x_{1}, \ldots, x_{n}\right]$ is graded (not necessarily standard graded). The notions of homogeneous ideal and degree are to be understood relative to this grading. If $M$ is graded then for any integer $d$ we write $M(d)$ for the $d$ th twist of $M$, i.e., the graded $A$-module with $M(d)_{i}=M_{i+d}$.

The following lemma follows immediately from additivity of dimension:

Lemma 5.1 (Lemma 5.1.2 in Greuel and Pfister (2002)) Let $A$ and $M$ be as above. Let $d$ be a nonnegative integer, $f \in A_{d}$ and $\varphi: M(-d) \rightarrow M$ be defined by $\varphi(m)=f \cdot m$; then $\operatorname{ker}(\varphi)$ and $\operatorname{coker}(\varphi)$ are graded $A /(f)$-modules with the induced gradings and

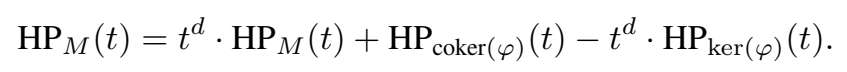

As an immediate consequence we obtain the useful:

Corollary 5.2 (Lemma 5.2.2 in Greuel and Pfister (2002)) Let $I \subseteq k\left[x_{1}, \ldots, x_{n}\right]$ be a homogeneous ideal, and let $f \in k\left[x_{1}, \ldots, x_{n}\right]$ be a homogeneous polynomial of degree $d$ then

$$
\mathrm{HP}_{k[x] / I}(t)=\operatorname{HP}_{k[x] /(I, f)}(t)+t^{d} \cdot \operatorname{HP}_{k[x] /(I: f)}(t) .
$$

For homogeneous ideals the leading ideal already determines the Hilbert-Poincaré series:

Theorem 5.3 (Theorem 5.2.6 in Greuel and Pfister (2002)) Let $>$ be a graded monomial ordering on a polynomial ring $k\left[x_{1}, \ldots, x_{n}\right]$, let $I \subseteq k[x]$ be a homogeneous ideal and denote by $L(I)$ its leading ideal with respect to $>$. Then

$$
\mathrm{HP}_{k[x] / I}(t)=\mathrm{HP}_{k[x] / L(I)}(t)
$$




\section{References}

G. Andrews. The theory of partitions. Cambridge Mathematical Library. Cambridge University Press, Cambridge, 1998. ISBN 0-521-63766-X. Reprint of the 1976 original.

G. Andrews and R. J. Baxter. A motivated proof of the Rogers-Ramanujan identities. Amer. Math. Monthly, 96(5):401-409, 1989. ISSN 0002-9890. doi: 10.2307/2325145. URL http://dx. doi. org/10.2307/2325145

C. Bruschek. Jet algebras and bell polynomials. Preprint, 2010.

C. Bruschek. The linearization principle in infinite dimensional algebraic geometry. PhD thesis, 2009.

C. Bruschek, H. Mourtada, and J. Schepers. Arc spaces and rogers-ramanujan identities. arXiv:1101.4950v2 [math.AG], 2011.

D. Cox, J. Little, and D. O'Shea. Ideals, varieties, and algorithms. Undergraduate Texts in Mathematics. Springer-Verlag, New York, second edition, 1997. ISBN 0-387-94680-2. An introduction to computational algebraic geometry and commutative algebra.

J. Draisma. Finiteness for the $k$-factor model and chirality varieties. Adv. Math., 223(1):243-256, 2010. ISSN 0001-8708. doi: 10.1016/j.aim.2009.08.008. URL http://dx.doi.org/10.1016/j . aim.2009.08.008.

L. Ein and M. Mustaţă. Inversion of adjunction for local complete intersection varieties. Amer. J. Math., 126(6):1355-1365, 2004. ISSN 0002-9327. URL http://muse. jhu.edu/journals/ american_journal_of_mathematics/v126/126.6ein.pdf.

B. Feigin and E. Frenkel. Coinvariants of nilpotent subalgebras of the Virasoro algebra and partition identities. In I. M. Gel'fand Seminar, volume 16 of Adv. Soviet Math., pages 139-148. Amer. Math. Soc., Providence, RI, 1993.

R. A. Goward, Jr. and K. E. Smith. The jet scheme of a monomial scheme. Comm. Algebra, 34(5):15911598, 2006. ISSN 0092-7872. doi: 10.1080/00927870500454927. URL http://dx.doi.org/ $10.1080 / 00927870500454927$.

G.-M. Greuel and G. Pfister. A Singular introduction to commutative algebra. Springer-Verlag, Berlin, 2002. ISBN 3-540-42897-6. With contributions by Olaf Bachmann, Christoph Lossen and Hans Schönemann, With 1 CD-ROM (Windows, Macintosh, and UNIX).

C. Hillar and S. Sullivant. Finite groebner bases in infinite dimensional polynomial rings and applications. http://arxiv.org/abs/0908.1777, 2009.

S. Ishii. Jet schemes, arc spaces and the Nash problem. C. R. Math. Acad. Sci. Soc. R. Can., 29(1):1-21, 2007. ISSN 0706-1994.

H. Mourtada. Sur les espaces des jets de quelques variétés algébriques singuliéres. PhD thesis, 2010.

J. Nash, Jr. Arc structure of singularities. Duke Math. J., 81(1):31-38 (1996), 1995. ISSN 0012-7094. A celebration of John F. Nash, Jr. 